\title{
A Comparison of Two Different Formulations For Arc Routing Problems on Mixed Graphs
}

\author{
Angel Corberán ${ }^{1 *}$, Enrique Mota ${ }^{1}$, José M. Sanchis $^{2}$ \\ ${ }^{1}$ Dept. d'Estadística i Investigació Operativa, Universitat de València, Spain \\ ${ }^{2}$ Dept. de Matemática Aplicada, Universidad Politécnica de Valencia, Spain
}

July 2003

\begin{abstract}
Arc Routing Problems on mixed graphs have been modelled in the literature either using just one variable per edge or associating to each edge two variables, each one representing its traversal in the corresponding direction. In this paper, and using the Mixed General Routing Problem as an example, we compare theoretical and computationally both formulations as well as the lower bounds obtained from them using Linear Programming based methods. Extensive computational experiments, including some big and newly generated random instances, are presented.
\end{abstract}

Key Words: Arc Routing, Mixed Chinese Postman Problem, Mixed Rural Postman Problem, Mixed General Routing Problem.

\section{Introduction}

Arc Routing Problems consist of finding a minimum cost set of routes over all or some of the links of a graph satisfying some side constraints. See the book edited by Dror (2000) for an excellent survey of the state of the art and applications of the field of Arc Routing. The basic problem, known as the Chinese Postman Problem $(C P P)$, is that of determining a minimum cost closed walk traversing at least once each edge of a non directed graph. From this basic and simple problem, the research, motivated by real-world applications, has focused along the last two decades on increasingly more difficult and general problems.

One step further in this generalization is to consider an underlying graph consisting both of edges and arcs. When the basic CPP is defined on such a mixed graph, the problem is known as the Mixed Chinese Postman Problem $(M C P P)$, and it is $N P$-hard, as shown by Papadimitriou (1976). Ford and Fulkerson (1962) were the first to present a characterization of those (Eulerian) mixed graphs that admit a closed walk traversing each link (edge or arc) exactly once. The MCPP was addressed by Christofides et al. (1984), who proposed a Branch \& Bound algorithm for its resolution. Using a similar formulation for a more general

\footnotetext{
${ }^{*}$ corresponding author: angel.corberan@uv.es
} 
problem, the Windy Postman Problem (WPP), Grötschel and Win (1992) designed a cuttingplane algorithm capable of solving medium size MCPP instances to optimality. Some years later, Nobert and Picard (1996), using a somewhat different formulation, presented another cutting-plane algorithm for this problem. This procedure is able to optimally solve instances of medium-large size. The MCPP is a special case of the Mixed Rural Postman Problem $(M R P P)$, which consists of finding a minimum cost closed walk traversing a subset of the links of a mixed graph; these links are then the required ones, while the non-required links may be part of the solution. If, in a more general context, a subset of vertices requiring service is also considered, we have the Mixed General Routing Problem ( $M G R P)$ that, although not strictly an arc routing problem, is one of the more general uncapacitated routing problems with a single vehicle and contains all the previous mentioned arc routing problems, as well as their undirected and directed versions, as special cases.

The MGRP has been recently studied in Corberán, Romero \& Sanchis (2003) and Corberán, Mejía \& Sanchis (2002). In the second paper a cutting-plane algorithm based on most of the known valid and facet-defining inequalities of the MGRP polyhedron is presented. This MGRP study starts from a formulation in which only one variable is associated to each edge. As the formulation proposed by Nobert \& Picard (1996) for the MCPP, it is based on the characterization of an Eulerian mixed graph given by Ford \& Fulkerson (1962). But some routing problems on mixed graphs have also been modelled using two variables associated to the same edge (see for example Christofides et al. (1984) and Grötschel and Win (1992)), each variable representing its traversal in the corresponding direction. In fact, during the revision of [5], an anonymous referee argued that formulating the MGRP using two variables for each edge would produce a stronger LP relaxation. To convince him/her, authors did some computational experiments that showed that the lower bounds obtained from the formulation using only one variable per edge were better than those obtained with the other one. But later on an error was detected in the coding of the cutting-plane algorithm based on the two variables per edge formulation. Once the mistake was corrected and the computational experiments repeated, the results were then not so conclusive. Therefore, from the above discussion, we think that this important point deserves to be studied in depth. This is the purpose of this paper.

We will compare these two alternative formulations to arc routing problems on mixed graphs choosing a very general problem, the MGRP, as an example. In Section 2 we define precisely the problem, introducing the notation to be used along the following sections and presenting the two different integer formulations of the MGRP, which are shown to be equivalent. In Section 3, and for each formulation, we obtain initial lower bounds from linear programming based methods. Although the initial LP relaxations are not equivalent, we obtain equivalent linear formulations once that every valid inequality from the classes that are known to be separated in polynomial time is also considered. Bounds obtained from both 'polynomial' linear relaxations can be further improved by adding other valid inequalities as they are (heuristically) detected. Section 4 presents the computational experiments in which both formulations are compared over an extensive set of newly generated instances. The last Section includes a brief summary and the conclusions of the research. 


\section{Problem Definition and Formulations}

Given a strongly connected mixed graph $G=(V, E, A)$ with vertex set $V$, edge set $E$, arc set $A$, a cost $c_{e}$ for each link $e \in E \cup A$, a set $E_{R} \subseteq E$ of required edges, a set $A_{R} \subseteq A$ of required arcs and a set $V_{R} \subseteq V$ of required vertices, the Mixed General Routing Problem (MGRP) is the problem of finding a minimum cost closed walk (a tour) passing through each required link $e \in E_{R} \cup A_{R}$ and through each $i \in V_{R}$ at least once.

Note that if $i \in V$ is a vertex incident to any required link $e \in E_{R} \cup A_{R}$, the condition on the tour passing through link $e$ contains the condition of visiting vertex $i$. Therefore, in what follows, we will assume that $V_{R}$ contains the set of vertices incident to the required edges. Furthermore, as it is usual when the subgraph induced by the required vertices and links is not connected, we first transform the original graph in order to simplify both the problem structure and the formulation. This transformation is done in a similar way to that of Christofides et al. (1981) for the undirected RPP (see Eiselt, Gendreau \& Laporte, 1995, for an illustration of the procedure). The graph resulting from this transformation is such that all its vertices are required ones as well as its edges, i.e., $V \backslash V_{R}=\emptyset$ and $E \backslash E_{R}=\emptyset$. Then, we will assume that the MGRP is defined on a strongly connected mixed graph $G=(V, E, A):=\left(V_{R}, E_{R}, A_{R} \cup A_{N R}\right)$.

We will use the same notation as in [5] and [4]. Let $G^{R}=G\left(V, E, A_{R}\right)$ be the graph obtained by deleting in $G$ all the non required arcs $A_{N R}$. In general, graph $G^{R}$ is not connected. Let $p$ be the number of connected components of $G^{R}$ and let $V_{1}, V_{2}, \ldots, V_{p}$ be the vertex sets corresponding to the $p$ connected components of $G^{R}$, which will be called $R$-sets, with $V_{1} \cup \ldots \cup V_{p}=V$. We will represent by $C_{i}=G\left(V_{i}\right), i=1, \ldots, p$, the subgraphs of $G$ induced by the $R$-sets and they will be referred to as $R$-connected components. Notice that every isolated required vertex is a R-connected component of $G$. Given two disjoint sets of vertices $S_{1}, S_{2} \subset V$ and a set $S \subset V$, we denote:

$$
\begin{aligned}
& \left(S_{1}: S_{2}\right)=\left\{(i, j) \in E \cup A: i \in S_{1}, j \in S_{2} \quad \text { or } \quad i \in S_{2}, j \in S_{1}\right\} \\
& \delta(S)=(S: V \backslash S) \text { (called link cut-set of } G \text { defined by } S) \\
& A^{+}(S)=A(S: V \backslash S) \\
& A^{-}(S)=A(V \backslash S: S) \\
& E(S)=E(S: V \backslash S)
\end{aligned}
$$

The above sets are defined in a similar way referring to required links only and to non-required arcs only: $A_{R}^{+}(S), A_{N R}^{-}(S), \delta_{R}(S)$, etc. Given $x \in \mathbb{R}^{|E \cup A|}$ and given $T \subset E \cup A, x(T)$ denotes $\sum_{e \in T} x_{e}$

A tour for the $M G R P$ is a family $\mathcal{F}$ of links of $G$ such that the graph $\left(V, E^{\mathcal{F}} \cup A^{\mathcal{F}}\right)$ contains all the required links and vertices and a closed walk traversing each copy of each link exactly once (i.e., is an Eulerian graph), where $E^{\mathcal{F}} \cup A^{\mathcal{F}}$ is obtained by considering each copy of a link in $\mathcal{F}$ as a different element.

Applying the necessary and sufficient conditions for a connected mixed graph to be Eulerian $([9])$, we can state that $\mathcal{F}$ is a tour for the MGRP when the following conditions are satisfied:

- $\mathcal{F}$ contains all the required links,

- $\operatorname{Graph}\left(V, E^{\mathcal{F}} \cup A^{\mathcal{F}}\right)$ is connected,

- Graph $\left(V, E^{\mathcal{F}} \cup A^{\mathcal{F}}\right)$ is even, i.e., every vertex is incident to an even number of links, 
- Graph $\left(V, E^{\mathcal{F}} \cup A^{\mathcal{F}}\right)$ is balanced, i.e., for every $S \subset V$, the difference between the number of arcs in $A^{+}(S)$ (leaving $S$ ) and the number of $\operatorname{arcs}$ in $A^{-}(S)$ (entering $S$ ) is less than or equal to the number of edges in $E(S)$ (balanced-set condition for set $S$ ).

The family of links obtained from any tour for the MGRP by deleting one copy of every link in $E \cup A_{R}$ is called a semitour for the MGRP. Although in [5] and [4] the MGRP is formulated with respect to semitours, in this paper and for the sake of simplicity, we will always consider MGRP tours. Associated to each MGRP tour there is an integer incidence vector $y=\left(y_{e}: \quad e \in E \cup A\right) \in \mathbb{R}^{|E \cup A|}$, where $y_{e}$ denotes the number of times a link $e \in E \cup A$ appears in the tour. If it is necessary to make an explicit reference to the direction in which a required link is traversed, we will use $y_{i j}$ instead of $y_{e}$. A vertex $v \in V$ will be called $R$-odd if it is incident to an odd number of required likes, otherwise it will be called $R$-even. Note that every isolated required vertex is R-even.

It is easy to see that the set of tours for the MGRP is then the set of vectors $y \in \mathbb{R}^{|E \cup A|}$ satisfying

$$
\begin{aligned}
y_{i j} \geq 0 \text { and integer, } & \forall(i, j) \in A_{N R} \\
y_{i j} \geq 1 \text { and integer, } & \forall(i, j) \in A_{R} \\
y_{e} \geq 1 \text { and integer, } & \forall e \in E_{R} \\
y(\delta(\{i\})) \equiv 0 \bmod 2, & \forall i \in V: i \text { is } R-\text { even } \\
y(\delta(\{i\})) \equiv 1 \bmod 2, & \forall i \in V: i \text { is } R-\text { odd } \\
y\left(A^{+}(S)\right) \geq 1, & \forall S=\cup_{k \in Q} V_{k}, Q \subset\{1, \ldots, p\} \\
y\left(A^{+}(S)\right)-y\left(A^{-}(S)\right) \leq y(E(S)), & \forall S \subset V
\end{aligned}
$$

where (2) and (3) imply that all the required links are in the solution, (4) and (5) and (6) assure that the resulting graph will be even and connected, respectively, and (7) that the balanced-set conditions will be satisfied. This formulation of the MGRP will be denoted by F1 and (with respect to semitours) was the one used in [5] and [4].

Consider now a set $S \subset V$ such that $E(S)=\emptyset$. Then the balanced-set condition corresponding to $S$ and $\bar{S}=V \backslash S$ imply the so called symmetry equation $y\left(A^{+}(S)\right)=y\left(A^{-}(S)\right)$. Hence, the above formulation includes an equation associated with each set $S \subset V$ with $E(S)=\emptyset$. Although most of these equations will be linearly dependent, if we consider the $q$ subgraphs of $G$ induced by the required edges, it can be shown that any $q-1$ of the corresponding symmetry equations are linearly independent. In [5] these equations

$$
y\left(A^{+}\left(K_{i}\right)\right)=y\left(A^{-}\left(K_{i}\right)\right), \quad i=1,2, \ldots, q
$$

are referred to as the system equations, where $K_{1}, K_{2}, \ldots, K_{q}$ denote the sets of vertices of the connected components of the graph $(V, E)$. Note that some sets $K_{i}$ could consist of a single vertex, that each set $K_{i}$ is contained in a set $V_{j}$ and that $E\left(V_{j}\right)=\emptyset, \forall j$.

Alternatively, if we choose the sufficient conditions for a mixed graph to be Eulerian ([9], $[7]), \mathcal{F}$ is a tour for the MGRP if

- $\mathcal{F}$ contains all the required links

- $\operatorname{Graph}\left(V, E^{\mathcal{F}} \cup A^{\mathcal{F}}\right)$ is connected,

- $\operatorname{Graph}\left(V, E^{\mathcal{F}} \cup A^{\mathcal{F}}\right)$ is even,

- Graph $\left(V, A^{\mathcal{F}}\right)$ is symmetric, i.e., the number of arcs entering every vertex is equal to the number of arcs leaving it. 
Note that if every vertex is symmetric, the number of arcs entering any $S \subset V$ should also be equal to the number of arcs leaving it.

The above conditions can be used to formulate the problem by associating two variables $x_{i j}$ and $x_{j i}$ to each edge $e=(i, j)$, representing the number of times edge $e$ is traversed in the corresponding direction. Then, the set of tours for the MGRP, according to this second formulation, called F2 in what follows, is the set of vectors $x \in \mathbb{R}^{2|E|+|A|}$ satisfying

$$
\begin{array}{rll}
x_{i j} \geq 0 \text { and integer, } & \forall(i, j) \in A_{N R} \\
x_{i j} \geq 1 \text { and integer, } & \forall(i, j) \in A_{R} \\
x_{i j}, x_{j i} \geq 0 \text { and integer, } \quad & \forall e=(i, j) \in E_{R} \\
& x_{i j}+x_{j i} \geq 1, \quad \forall e=(i, j) \in E_{R} \\
& x\left(A^{+}(S)\right) \geq 1, \quad \forall S=\cup_{k \in Q} V_{k}, \quad Q \subset\{1, \ldots, p\} \\
x\left(A^{+}(i)\right)+\sum_{j:(i, j) \in E_{R}} x_{i j}=x\left(A^{-}(i)\right)+\sum_{j:(i, j) \in E_{R}} x_{j i}, \quad \forall i \in V
\end{array}
$$

where (10) and (12) imply that all the required links are in the solution, (13) assure that the resulting graph will be connected and (14) that the symmetry conditions will be satisfied. Note that integrality and symmetry conditions imply that the resulting graph will be even.

In what follows, we show that formulations F1 and F2 are equivalent. Let $x$ be a solution to F2 and define $y_{e}=x_{i j}+x_{j i}, \forall e=(i, j) \in E_{R}$ and $y_{i j}=x_{i j}, \forall(i, j) \in A$. Then, constraints (9)-(12) imply constraints (1)-(3). Furthermore, since $x$ induces a connected and symmetric graph, $y$ defines an even and connected graph. And given that every vertex is symmetric with respect to $x$, a symmetry equation holds for each cut $(S: V \backslash S)$ and, therefore, the balancedset condition with respect to $y$ is satisfied for each $S$. Thus, from any feasible solution to F2 we can obtain a feasible solution to F1 with the same cost. Conversely, consider now a solution to F1: it induces an even, connected and balanced mixed graph. Edmonds and Johnson (1973) proposed a method of assigning a direction to the edges of an even and balanced mixed graph in order to obtain, with the same cost, a symmetric directed graph. Thus, from any feasible solution to F1 we can obtain a feasible solution to F2 with the same cost.

\section{Lower Bounds from Linear Programming Based Methods}

Once we have proved that both (integer) formulations are equivalent, we are now interested in comparing them in order to obtain lower bounds for the MGRP using Linear Programming based methods. To do this the integrality conditions in F1 and F2 must be removed. Furthermore, constraints (4) and (5) in F1 are not linear and there is not a linear expression of them without using integer variables. Hence, these constraints should also be removed. However, it is easy to see that for each R-odd vertex $i, y(\delta(i)) \geq\left|\delta_{R}(i)\right|+1$ must hold. In a similar way, given an $R$-odd cutset $\delta(S)$ (a cutset containing an odd number of required links), the following inequalities, called $R$-odd cut inequalities, must be satisfied by any solution $y$ to F1 (see [5])

$$
y(\delta(S)) \geq\left|\delta_{R}(S)\right|+1, \forall \delta(S) \quad R \text {-odd cutset }
$$


On the other hand, once the integrality conditions have been removed from F2, symmetry conditions (14) alone do not longer guarantee that all the vertices will be even. However, constraints (15) should also be satisfied by any solution $x$ to F2.

Therefore, we will call $\mathrm{LP}_{F 1}$ the following linear relaxation of $\mathrm{F} 1$

$$
\begin{aligned}
y_{i j} \geq 0, & \forall(i, j) \in A_{N R} \\
y_{i j} \geq 1, & \forall(i, j) \in A_{R} \\
y_{e} \geq 1, & \forall e \in E_{R} \\
y\left(A^{+}(S)\right) \geq 1, & \forall S=\cup_{k \in Q} V_{k}, \quad Q \subset\{1, \ldots, p\} \\
y\left(A^{+}(S)\right)-y\left(A^{-}(S)\right) \leq y(E(S)), & \forall S \subset V \\
y(\delta(S)) \geq\left|\delta_{R}(S)\right|+1, & \forall \delta(S) \quad R \text {-odd cutset }
\end{aligned}
$$

and $\mathrm{LP}_{F 2}$ the following linear relaxation of $\mathrm{F} 2$

$$
\begin{aligned}
& x_{i j} \geq 0, \forall(i, j) \in A_{N R} \\
& x_{i j} \geq 1, \forall(i, j) \in A_{R} \\
& x_{i j}, x_{j i} \geq 0, \forall e=(i, j) \in E_{R} \\
& x_{i j}+x_{j i} \geq 1, \forall e=(i, j) \in E_{R} \\
& x\left(A^{+}(S)\right) \geq 1, \forall S=\cup_{k \in Q} V_{k}, \quad Q \subset\{1, \ldots, p\} \\
& x(\delta(S)) \geq\left|\delta_{R}(S)\right|+1, \forall \delta(S) \quad R \text {-odd cutset } \\
& x\left(A^{+}(i)\right)+\sum_{j:(i, j) \in E_{R}} x_{i j}=x\left(A^{-}(i)\right)+\sum_{j:(i, j) \in E_{R}} x_{j i}, \quad \forall i \in V
\end{aligned}
$$

\subsection{Initial Lower Bounds}

Note that constraints (19)-(21) in $\mathrm{LP}_{F 1}$ and (26)-(27) in $\mathrm{LP}_{F 2}$ are exponential in number. Therefore, only a subset of them can be explicitly added to the LP's to be solved. As in [5] and [4], we define the following initial $\mathrm{LP}$ relaxation, $\mathrm{LP}_{F 1}$, corresponding to $\mathrm{LP}_{F 1}$ containing

- the system equations (8)

- one connectivity inequality (19) for each $R$-set

- one balanced-set inequality (20) for each 'unbalanced' vertex

- one $R$-odd cut inequality (21) for each $R$-odd 'balanced' vertex,

where an unbalanced vertex $i$ is a vertex for which $|E(i)|<\left|A_{R}^{+}(i)\right|-\left|A_{R}^{-}(i)\right|$ or $|E(i)|<$ $\left|A_{R}^{-}(i)\right|-\left|A_{R}^{+}(i)\right|$.

In a similar way we define $\mathrm{LP}_{F 2}$ (corresponding to $\mathrm{LP}_{F 2}$ ) as the $\mathrm{LP}$ containing

- one connectivity inequality (26) for each $R$-set

- one $R$-odd cut inequality (27) for each $R$-odd 'balanced' vertex

- one symmetry equation (28) for each vertex. 
In what follows, we show that $\mathrm{LP} 0_{F 2}$ is stronger that $\mathrm{LP}_{F 1}$. Consider a feasible solution $x$ to $\mathrm{LP}_{F 2}$ and define again $y_{e}=x_{i j}+x_{j i}, \forall e=(i, j) \in E_{R}$ and $y_{i j}=x_{i j}, \forall(i, j) \in A$. If $x$ satisfies the symmetry constraints (28) for every vertex, $y$ satisfies all the system equations and also the balanced-set constraints (20) for each vertex. Then, from any feasible solution to $\mathrm{LP}_{F 2}$ we obtain a feasible solution to $\mathrm{LP}_{F 1}$ with the same cost. However, the converse is not true, as the following example shows. The graph in Figure 1, in which all the links are required, represents a feasible solution to $\mathrm{LP}_{F 1}$ (although the balanced-set conditions are not satisfied for every subset $S$ of vertices). However, there is no way of directing the edges so as, with the same cost, obtaining a symmetric graph. Therefore, the lower bound obtained using $\mathrm{LP}_{F 1}$ cannot be greater than the lower bound obtained using $\mathrm{LP} 0_{F 2}$. This fact is reflected in column LB0 in the tables in Section 4.

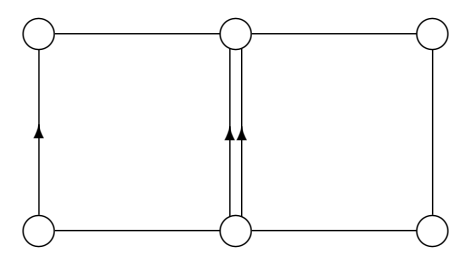

Figure 1: Feasible solution to $\mathrm{LP} 0_{F 1}$

\subsection{Lower Bounds in Polynomial Time}

Since there is, as already mentioned, an exponential number of inequalities in some of the families defining $\mathrm{LP}_{F 1}$ and $\mathrm{LP}_{F 2}$, not all of them can be explicitly included in a $\mathrm{LP}$ to be solved. One alternative in order to solve these programs is to use the following iterative process, called cutting-plane algorithm. To begin with, compute the solution of an LP consisting of a small subset of inequalities (in our case, those in $\mathrm{LP} 0_{F 1}$ and $\mathrm{LP}_{F 2}$ ). Then, look for inequalities, not in the current LP, that are violated by its optimal solution. If one or more violated inequalities are detected, add them to the current LP and solve it again; otherwise, terminate. This process needs to solve the 'separation problem', i.e., the identification of those inequalities that are violated by the current LP solution. This problem is solved in practice using a different algorithm for each class of valid inequalities. An exact separation algorithm for a given class of inequalities is a routine that, having an LP solution as input, outputs one or more violated inequalities in that class, whenever there are violated inequalities. A heuristic separation algorithm works in a similar way, but it may fail to detect violated inequalities in the class.

The separation problems associated with connectivity and $R$-odd cut inequalities are solvable in polynomial time by means of max-flow calculations and the Padberg \& Rao (1982) procedure to find minimum odd cut-sets. Nobert \& Picard (1996) showed that identifying violated balanced-set inequalities for the MCPP can also be solved in polynomial time. This result is easily extended to the MGRP. Therefore, $\mathrm{LP}_{F 1}$ and $\mathrm{LP}_{F 2}$ can be solved in polynomial time (assuming that a Linear Programming polynomial time algorithm is used).

In what follows, we will show that these two linear problems are equivalent. As before, it is easy to see that the set of feasible solutions to $\mathrm{LP}_{F 2}$ is included in that of $\mathrm{LP}_{F 1}$. Consider now a (possibly fractional) feasible solution $y$ to $\mathrm{LP}_{F 1}$. Let $G_{y}$ be the weighted 
graph with vertex set $V$, link set $\left\{e \in E \cup A: y_{e}>0\right\}$ and link-weights $y_{e}$. The question is how to assign a direction to the edges of $G_{y}$ in order to obtain a directed graph satisfying the symmetry equations. To do this, we first solve a flow problem on an undirected graph $H=\left(V_{H}, E_{H}\right)$, where $V_{H}=V \cup\{0, n+1\}$ ( 0 and $n+1$ are two extra vertices). The set of edges $E_{H}$ includes all the edges in $E$, with an associated capacity $y_{e}$, plus one edge from vertex 0 to every vertex $i \in V$ with capacity $y_{0 i}=\max \{d(i), 0\}$, where $d(i)=y\left(A^{-}(i)\right)-y\left(A^{+}(i)\right)$, and one edge from each $i \in V$ to vertex $n+1$ with capacity $y_{i, n+1}=\max \{-d(i), 0\}$. Let $P=\sum_{d(i)>0} d(i)=\sum_{d(i)<0}-d(i)$

Compute a maximum flow in $H$ from vertex 0 to vertex $n+1$. Since $y$ satisfies all the balanced-set inequalities, the flow equals $P$ (otherwise, as it is shown in [1], a balanced-set inequality violated by $y$ would exist). Hence, this implies that it is possible to satisfy all the supplies $(d(i)>0)$ and demands $(d(i)<0)$ of the vertices in $G_{y}$, computed with respect to its arcs, using only its edges.

Now, let $G(A)$ be the weighted graph induced by the arcs in $G_{y}$. From the optimal solution $f$ to the above flow problem,

- if the optimal flow through a given edge $e$ is 0 , add to $G(A)$ two arcs, in opposite directions, each one with weight $y_{e} / 2$.

- if the optimal flow through an edge equals its capacity, add to $G(A)$ one arc with weight $y_{e}$ in the direction given by the flow.

- if the optimal flow $f_{e}$ through an edge $e=(i, j)$ is less than its capacity, add to $G(A)$ one arc in the direction given by the flow, with weight $f_{e}$. Add also two more $\operatorname{arcs}(i, j)$ and $(j, i)$ with weights equal to $\left(y_{e}-f_{e}\right) / 2$.

The resulting graph is symmetric and has the same cost as $G_{y}$. Note that the above process is equivalent to construct a feasible solution $x$ to $\mathrm{LP}_{F 2}$ by defining $x_{i j}=y_{i j}$ for all $\operatorname{arcs}(i, j)$ in $A$ and $x_{i j}=\left(y_{e}+f_{e}\right) / 2$ and $x_{j i}=\left(y_{e}-f_{e}\right) / 2$ for every edge $e=(i, j)$, if $(i, j)$ is the direction given by the flow. Therefore, from a feasible solution $y$ to $\mathrm{LP}_{F 1}$ we have obtained a feasible solution $x$ to $\mathrm{LP}_{F 2}$ with the same cost.

Hence, $\mathrm{LP}_{F 1}$ and $\mathrm{LP}_{F 2}$ are equivalent and the bounds obtained from them (column LBpol in tables) are equal, as it can also be seen from the computational experiments.

\subsection{Improving the Bounds}

In the previous Section, the cutting-plane algorithm uses only the inequalities that appear in $\mathrm{LP}_{F 1}$ and $\mathrm{LP}_{F 2}$. Furthermore, these inequalities belong to classes that can be separated in polynomial time. However, the cutting-plane methodology allows the addition of any other valid inequality (one that is satisfied by all the feasible solutions) in order to improve the bound. The polyhedron associated to the MGRP solutions defined by F1, MGRP $(G)$, has been studied in [4] and [5]. Besides connectivity, R-odd cut and balanced-set inequalities, other families of facet-inducing inequalities are also described: (standard) $K-C, K-C_{02}$, (standard) Honeycomb and Honeycomb $b_{02}$.

Since no exact polynomial algorithm is known for the separation problems of all these latter inequalities, in the cutting-plane procedure described in [4], heuristic procedures were 
used to find violated inequalities of these classes. This cutting-plane algorithm has also been used here in order to get a better lower bound. Hence, in what follows we will represent by $\mathrm{LB}_{F 1}$ the lower bound obtained using the cutting-plane procedure described in [4].

Although the polyhedron associated to the MGRP solutions defined by F2 has not been explicitly studied, it should share most of the characteristics associated to polyhedron $\operatorname{MGRP}(G)$. Consider a feasible solution $x$ to F2. As before, by adding the two $x$-variables corresponding to each edge $e=(i, j)$, we obtain a solution $y$ to F1. Solution $y$ should satisfy all the valid inequalities known for $\operatorname{MGRP}(G)$. Let $F(y)=\sum c_{e} y_{e} \geq b$ be such an inequality. It is easy to see that the inequality $F(x)=\sum c_{e}\left(x_{i j}+x_{j i}\right) \geq b$ is valid for $x$. Then, standard $\mathrm{K}-\mathrm{C}, \mathrm{K}-\mathrm{C}_{02}$, standard Honeycomb and Honeycomb ${ }_{02}$ inequalities can be slightly modified in order to be valid inequalities for the MGRP solutions defined by F2. The corresponding separation procedures have been also modified and used to obtain a better lower bound $\mathrm{LB}_{F 2}$ by means of the cutting-plane algorithm based on formulation F2.

It is not known wether the inequalities mentioned in this section can or not be separated in polynomial time, but our guess is that these problems are $\mathcal{N} \mathcal{P}$-hard. The use of heuristic algorithms to find violated inequalities of these classes implies that, although from a theoretical point of view bounds $\mathrm{LB}_{F 1}$ and $\mathrm{LB}_{F 2}$ could be equal, in practice they can provide different values. The computational results shown in the next section confirm this fact.

\section{Computational Testing}

In this Section we present the computational experiments conducted and the results obtained using the cutting-plane algorithms described in the previous sections.

\subsection{The Instances}

In order to study in depth the differences between the two formulations, we have generated several sets of instances corresponding to some of the more important arc routing problems on mixed graphs, the MCPP, MRPP and MGRP. Let us begin with a description of the procedure used to generate these sets of instances.

On one hand, we have used graphs modelling the real street network of the Spanish towns of Albaida, Madrigueras and Aldaya. Graphs of Albaida and Madrigueras are undirected graphs having 116 vertices and 174 edges and 196 vertices and 316 edges, respectively. Although the Aldaya real street network corresponds to a mixed graph having 214 vertices and 224 edges and 127 arcs, here it will be considered as an undirected graph with 351 edges.

From these graphs, we first generate MCPP instances in the following way. All the edges in $E$ are read in sequential order and some of them are transformed into an arc with a given probability $p$. If edge $(i, j)$ is selected to be transformed into an arc, an orientation $(i, j)$ or $(j, i)$ is assigned with probability 0.5. Finally, if the number of strongly connected components is greater than one, some arcs are transformed back into edges to obtain a strongly connected graph. From each graph (Albaida, Madrigueras and Aldaya) and for each value of $p \in\{0.3,0.5,0.7\}$ we have generated three different instances. As an example, albaC3A, albaC3B and albaC3C are the three corresponding to the Albaida graph and $p=0.3$ (' $\mathrm{C}$ ' 
means Chinese). Then, we have 27 instances for the MCPP.

A similar process is used to generate instances for the MRPP and for the MGRP. Again, each edge is transformed into an arc with probability $p \in\{0.3,0.5,0.7\}$ and labelled as 'required' with probability $q \in\{0.3,0.5,0.7\}$. At this point we have an instance for the MGRP if we consider all the vertices as 'required'. In order to obtain an instance for the MRPP in which all the vertices are incident with required links, we iteratively select a link $e$ with one end-point that is not incident with a 'required' link and label it as 'required' with the same probability $q$. This second step is repeated until all the vertices in $V$ are incident with, at least, one 'required' link. From each graph (Albaida, Madrigueras and Aldaya), and for each value of parameters $p \in\{0.3,0.5,0.7\}$ and $q \in\{0.3,0.5,0.7\}$, we have generated 27 instances, named albaR33 to aldaR77 ('R' means Rural), for the MRPP and 27 other instances, named albaG33 to aldaG77 ('G' means General), for the MGRP.

On the other hand, in order to test the bounds on larger MRPP and MGRP instances, we decided to generate new random graphs. We first randomly selected 500 vertices in a $1000 \times 1000$ square. Edges were then generated as pairs of vertices $i$ and $j$, with costs defined by $c_{i j}=\left\lfloor b_{i j}+\frac{1}{2}\right\rfloor$, where $b_{i j}$ are the Euclidean distances. This is the same cost function proposed in the TSPLIB (see [14]). In order to obtain graphs with a structure similar to that of real street networks, we did not generate edges completely at random. Indeed, we proceeded as follows. Let $d$ (degree) be a parameter. Add an edge from each vertex $i$ to its $d$ nearest vertices. Then remove those edges $(i, j)$ for which $c_{i j} \geq 0.98\left(c_{i k}+c_{k j}\right)$, for some $(i, k)$ and $(k, j)$. Some of the remaining edges are then considered as 'required' with a given probability $q$. If the resulting graph is non connected, edges in the $d$ shortest trees spanning the connected components are also added and labelled as 'non required'. At this point and as before, if we consider all the vertices as 'required', we have an undirected GRP instance. Otherwise, to obtain an undirected RPP instance, the simplification procedure mentioned in Section 2 is applied. In both cases, from the undirected graphs, mixed instances are obtained as described above, where edges are transformed into arcs using a given probability $p$. By combining values $\{4,5\}$ for parameter $d$ and values $\{0.25,0.50,0.75\}$ for parameters $q$ and $p$, we have generated 18 MRPP instances, named R422 (meaning 'Rural', $d=4, q=0.25$, $p=0.25)$ to R577 and 18 MGRP instances, named G422 to G577.

\subsection{Computational Results}

The algorithms have been coded in $C$ and ran on a PC with a $2000 \mathrm{MHz}$. Pentium IV processor. Tables 1 to 11 show the computational results obtained for the instances described above.

All the tables contain two rows for each instance, presenting the results obtained from formulations $\mathrm{F} 1$ and $\mathrm{F} 2$. Columns labelled ' $p$ ', ' $V_{R}$ ' and ' $E \cup A$ ' represent, respectively, the number of $R$-sets, the number of (required) vertices and the number of links of each instance. Column 'LB0' shows the initial lower bound (see Section 3.1). Columns 'LBpol' and 'Tpol' present the bound obtained with the cutting-plane algorithm using only the inequalities that are known to be separated in polynomial time (see Section 3.2) and the time used in seconds, respectively. Next columns 'LB' and 'Time' give, respectively, the lower bound and the corresponding time in seconds obtained with the cutting-plane algorithms described in Section 3.3. These two last columns are not shown for the MCPP instances because the 
additional valid inequalities used to obtain the final bound LB do not apply when all the links are required. An '*' means than an optimal solution (not only the optimal value) has been obtained. Finally, column 'Dev' shows the percentage deviation of the LB bound from the optimal value. Note that when a cutting-plane algorithm finishes before the optimal solution is reached, we can resort to the branch-and-bound option of CPLEX. If the integer solution is a feasible solution, it is optimal. Otherwise, some inequalities violated by the integer solution can be detected and added to the last LP and the process continues. This is the procedure that, when needed, has been applied here to obtain the optimal solutions.

From the 27 MCPP instances reported in Tables 1 to 3 it is clear that the initial lower bound (LB0) corresponding to F2 is much better than that of F1, as it should be the case, since it has been proved that $\mathrm{LP}_{F 2}$ is stronger than $\mathrm{LP}_{F 1}$. Moreover, the corresponding polynomial bounds (LBpol) are obviously equal, although the cutting-plane procedure based on $\mathrm{F} 1$ is in general more time consuming. In any case, both algorithms obtain the optimal solutions of these medium-size instances within a few seconds.

The above comments are also valid for the MRPP instances in Table 4 to 6 . Note also that the polynomial lower bounds from F1 and F2 equal here the optimal values in 20 out of the 27 instances and that the final bound LB is better than LBpol since, within similar computing times, it obtains the previous 20 optimal values and 3 new ones. As expected, bounds LB from formulations F1 and F2 are similar. Finally, with respect to the times needed to compute LBpol and LB, note that both algorithms require a similar computational effort.

With respect to the MGRP instances in Tables 7 to 9 note that, when bound LBpol does not reach an optimal solution, the final bound LB is better than bound LBpol and that the improvement is similar with both formulations.

In order to get a deeper understanding of the differences between the two formulations, we decided to generate larger MRPP and MGRP instances, as described above. Some of the generated instances are really big, having up to $245 R$-sets, 500 vertices and 1339 links. We think that these are the biggest instances published so far.

To our surprise, the polynomial cutting-plane algorithms are able to optimally solve 9 out of the 18 MRPP instances and 6 out of the 18 MGRP instances, but these are those instances having the smaller number of $R$-sets. Note that in order to solve these instances it would not be necessary to look for inequalities that up to now can not be separated in polynomial time. However, if we include (through heuristics) some of these inequalities, as the final bound LB does, the optimal solution is also obtained with usually less computational effort and in some cases this effort is greatly reduced. The final bound LB improves on the polynomial bound LBpol in those instances for which the latter can not reach the optimal solution.

Finally, as mentioned at the end of Section 3.3, there are some differences between $\mathrm{LB}_{F 1}$ and $\mathrm{LB}_{F 2}$ but, as we expected, they are minimal. The small differences reported may be just due to differences in the sequence of the LP solutions found. With respect to the computing times, note that the LP's solved by the cutting-plane algorithm based on formulation F1 are smaller, since only one variable per edge is defined in F1, while the algorithm based on F2 does not require the identification of balanced-set inequalities at each iteration. Although on most of the instances the second algorithm terminates earlier, there are big differences between the effort taken by each algorithm on some of them. For example, in instances G422 and G522 in which the number of $R$-sets and required edges is big, the algorithm based on 
F1 is faster than that based on F2.

\section{Conclusions}

We summarize in this final section the conclusions of the comparison between the two proposed formulations for arc routing problems on mixed graphs. With respect to the initial bounds, it has been shown that $\mathrm{LP}_{F 2}$ dominates the initial bound obtained from formulation F1. Furthermore, the former can be easily obtained and the computational results show that it is much better than bound $\mathrm{LP}_{F 1}$. Then, if we are interested in a simple and good bound in order to test, for example, the quality of a feasible solution provided by a heuristic, we think that bound $\mathrm{LP}_{F 2}$ should be our first choice.

Polynomial bounds LBpol obtained from formulations F1 and F2 have been shown to be equal, though the algorithm based on F2 is usually faster since it only needs to identify inequalities in two classes.

As for the inequalities included in the bound, it pays to look (heuristically) for K-C, K$\mathrm{C}_{02}$, Honeycomb and Honeycomb 02 inequalities, since in both formulations the final bound LB improves on the polynomial bound, taking into account the values obtained and the computational times.

Bounds $\mathrm{LB}_{F 1}$ and $\mathrm{LB}_{F 2}$ obtained using the cutting-plane procedures from formulations F1 and F2 are generally equal. With respect to the algorithms providing them, we would like to point to two main differences. While, on one hand, the algorithm based on F1 deals with only one variable per edge and therefore the LP's to be solved are smaller, that based on F2 does not require the identification of balanced-set inequalities at each iteration and therefore

it is easier to code and should be faster. However, although in some instances this latter algorithm terminates earlier, there is no a common trend with respect to the time needed to compute $\mathrm{LB}_{F 1}$ and $\mathrm{LB}_{F 2}$. Indeed, there are big differences between the effort taken by each algorithm on some instances.

Finally, as mentioned in Section 4.2, when a cutting-plane algorithm finishes before the optimal solution is reached, it is possible to invoke the branch-and-bound option of CPLEX in order to get an integer solution. In this case, an advantage of using formulation F2 is that when the integer solution is connected it is an optimal solution. This is not true if we use formulation F1, since in this case the integer solution should also be even and balanced.

\section{Acknowledgment}

Authors thank the support given by the Ministerio de Ciencia y Tecnología of Spain through grant TIC2000-C06-01. 


\section{References}

[1] E. Benavent, A. Corberán \& J.M. Sanchis (2000): "Linear Programming Based Methods for Solving Arc Routing Problems". In M. Dror (Ed.) Arc Routing: Theory, Solutions and Applications. Kluwer Academic Publishers.

[2] N. Christofides, E. Benavent, V. Campos, A. Corberán \& E. Mota (1984): “An optimal method for the Mixed Postman Problem". In P. Thoft-Christensen (Ed.) System Modelling and Optimization. Lecture Notes in Control and Information Sciences, 59. Berlin: Springer-Verlag.

[3] N. Christofides, V. Campos, A. Corberán \& E. Mota (1981): "An Algorithm for the Rural Postman Problem". Report IC.OR. 81.5. Imperial College, London.

[4] A. Corberán, G. Mejía \& J.M. Sanchis (2002): "New Results on the Mixed General Routing Problem". TR05-2002. Dept. d'Estadística i Investigació Operativa, Universitat de València. To appear in Operations Research.

[5] A. Corberán, A. Romero \& J.M. Sanchis (2003): “The Mixed General Routing Problem Polyhedron". Mathematical Programming, 96, 103-137.

[6] M. Dror (Ed.) (2000): Arc Routing: Theory, Solutions and Applications. Kluwer Academic Publishers.

[7] J. Edmonds \& E. L. Johnson (1973): "Matching, Euler Tours and the Chinese Postman". Mathematical Programming, 5, 88-124.

[8] H.A. Eiselt, M. Gendreau \& G. Laporte (1995): "Arc-Routing Problems, Part 2: the Rural Postman Problem". Operations Research, 43, 399-414.

[9] L.R. Ford \& D.R. Fulkerson (1962) Flows in Networks. Princeton University Press, Princeton, NJ.

[10] M. Grötschel \& Z. Win (1992): "A cutting-plane algorithm for the Windy Postman Problem". Mathematical Programming, 55, 339-358.

[11] Y. Nobert \& J.C. Picard (1996): "An optimal algorithm for the Mixed Chinese Postman Problem". Networks, 27, 95-108.

[12] M.W. Padberg \& M.R. Rao (1982) Odd minimum cut-sets and b-matchings. Math. Oper. Res., 7, 67-80.

[13] C.H. Papadimitriou (1976): "On the complexity of edge traversing". J. of the A.C.M., 23, 544-554.

[14] G. Reinelt (1991): "TSPLIB: a Traveling Salesman Problem Library". ORSA Journal of Computing 3, 376-384. 


\begin{tabular}{|c|c|c|c|c|c|c|c|}
\hline & $p$ & $V_{R}$ & $E \cup A$ & LB0 & LBpol & Tpol & $\operatorname{Dev}(\%)$ \\
\hline $\begin{array}{l}\text { albaC3A F1 } \\
\text { albaC3A F2 } \\
\text { albaC3B F1 } \\
\text { albaC3B F2 } \\
\text { albaC3C F1 } \\
\text { albaC3C F2 }\end{array}$ & 1 & 116 & 174 & $\begin{array}{l}14800 \\
15575 \\
14990 \\
16306 \\
15310 \\
18834\end{array}$ & $\begin{array}{l}16380^{*} \\
16380^{*} \\
17256^{*} \\
17256^{*} \\
19518^{*} \\
19518^{*}\end{array}$ & $\begin{array}{l}4.4 \\
5.8 \\
6.6 \\
6.8 \\
5.3 \\
4.1\end{array}$ & \\
\hline $\begin{array}{l}\text { albaC5A F1 } \\
\text { albaC5A F2 } \\
\text { albaC5B F1 } \\
\text { albaC5B F2 } \\
\text { albaC5C F1 } \\
\text { albaC5C F2 }\end{array}$ & 1 & 116 & 174 & $\begin{array}{l}19336 \\
23882 \\
15754 \\
17444 \\
17245 \\
20914\end{array}$ & $\begin{array}{l}24568^{*} \\
24568^{*} \\
18216^{*} \\
18216^{*} \\
21340^{*} \\
21340^{*}\end{array}$ & $\begin{array}{l}5.3 \\
1.6 \\
2.1 \\
1.9 \\
2.1 \\
1.9\end{array}$ & \\
\hline $\begin{array}{l}\text { albaC7A F1 } \\
\text { albaC7A F2 } \\
\text { albaC7B F1 } \\
\text { albaC7B F2 } \\
\text { albaC7C F1 } \\
\text { albaC7C F2 }\end{array}$ & 1 & 116 & 174 & $\begin{array}{l}16715 \\
18906 \\
20430 \\
25076 \\
16183 \\
20047\end{array}$ & $\begin{array}{l}19328^{*} \\
19328^{*} \\
25462^{*} \\
25462^{*} \\
20560^{*} \\
20560^{*}\end{array}$ & $\begin{array}{l}1.4 \\
1.3 \\
1.1 \\
0.7 \\
3.1 \\
1.9\end{array}$ & \\
\hline
\end{tabular}

Table 1: MCPP instances from Albaida graph

\begin{tabular}{|c|c|c|c|c|c|c|c|}
\hline & $p$ & $V_{R}$ & $E \cup A$ & LB0 & LBpol & Tpol & $\operatorname{Dev}(\%)$ \\
\hline $\begin{array}{l}\text { madrC3A F1 } \\
\text { madrC3A F2 } \\
\text { madrC3B F1 } \\
\text { madrC3B F2 } \\
\text { madrC3C F1 } \\
\text { madrC3C F2 }\end{array}$ & 1 & 196 & 316 & $\begin{array}{l}28165 \\
31110 \\
28245 \\
30580 \\
29385 \\
34730\end{array}$ & $\begin{array}{l}31610^{*} \\
31610^{*} \\
31115^{*} \\
31115^{*} \\
35140^{*} \\
35140^{*}\end{array}$ & $\begin{array}{r}19 \\
1.8 \\
15 \\
5.4 \\
14 \\
3.2\end{array}$ & \\
\hline $\begin{array}{l}\text { madrC5A F1 } \\
\text { madrC5A F2 } \\
\text { madrC5B F1 } \\
\text { madrC5B F2 } \\
\text { madrC5C F1 } \\
\text { madrC5C F2 }\end{array}$ & 1 & 196 & 316 & $\begin{array}{l}31445 \\
37820 \\
32605 \\
40975 \\
31780 \\
39435\end{array}$ & $\begin{array}{l}38355^{*} \\
38355^{*} \\
41085^{*} \\
41085^{*} \\
39730^{*} \\
39730^{*}\end{array}$ & $\begin{array}{l}9.1 \\
1.8 \\
6.7 \\
0.9 \\
9.5 \\
0.9\end{array}$ & \\
\hline $\begin{array}{l}\text { madrC7A F1 } \\
\text { madrC7A F2 } \\
\text { madrC7B F1 } \\
\text { madrC7B F2 } \\
\text { madrC7C F1 } \\
\text { madrC7C F2 }\end{array}$ & 1 & 196 & 316 & $\begin{array}{l}40505 \\
52572.5 \\
37120 \\
44360 \\
34445 \\
40790 \\
\end{array}$ & $\begin{array}{l}52735^{*} \\
52735^{*} \\
44540^{*} \\
44540^{*} \\
41210^{*} \\
41210^{*}\end{array}$ & $\begin{array}{l}3.3 \\
0.5 \\
4.3 \\
0.7 \\
5.0 \\
3.4 \\
\end{array}$ & \\
\hline
\end{tabular}

Table 2: MCPP instances from Madrigueras graph 


\begin{tabular}{|c|c|c|c|c|c|c|c|}
\hline & $p$ & $V_{R}$ & $E \cup A$ & LB0 & LBpol & Tpol & Dev $(\%)$ \\
\hline $\begin{array}{l}\text { aldaC3A F1 } \\
\text { aldaC3A F2 } \\
\text { aldaC3B F1 } \\
\text { aldaC3B F2 } \\
\text { aldaC3C F1 } \\
\text { aldaC3C F2 }\end{array}$ & 1 & 214 & 351 & $\begin{array}{l}41966 \\
43230 \\
42508 \\
46623.5 \\
42101 \\
46824.5\end{array}$ & $\begin{array}{l}44422^{*} \\
44422^{*} \\
47480^{*} \\
47480^{*} \\
47344^{*} \\
47344^{*}\end{array}$ & $\begin{array}{r}10 \\
6.1 \\
13 \\
3.6 \\
9.8 \\
2.1\end{array}$ & \\
\hline $\begin{array}{l}\text { aldaC5A F1 } \\
\text { aldaC5A F2 } \\
\text { aldaC5B F1 } \\
\text { aldaC5B F2 } \\
\text { aldaC5C F1 } \\
\text { aldaC5C F2 }\end{array}$ & 1 & 214 & 351 & $\begin{array}{l}45680 \\
53508 \\
52108 \\
63708 \\
48595 \\
55280.5\end{array}$ & $\begin{array}{l}54143^{*} \\
54143^{*} \\
64108^{*} \\
64108^{*} \\
55999^{*} \\
55999^{*}\end{array}$ & $\begin{array}{r}6.9 \\
1.1 \\
14 \\
1.5 \\
5.7 \\
0.9\end{array}$ & \\
\hline $\begin{array}{l}\text { aldaC7A F1 } \\
\text { aldaC7A F2 } \\
\text { aldaC7B F1 } \\
\text { aldaC7B F2 } \\
\text { aldaC7C F1 } \\
\text { aldaC7C F2 }\end{array}$ & 1 & 214 & 351 & $\begin{array}{l}55272 \\
64197 \\
66289 \\
77591 \\
53780 \\
66681.5\end{array}$ & $\begin{array}{l}64479^{*} \\
64479^{*} \\
77849^{*} \\
77849^{*} \\
67013^{*} \\
67013^{*}\end{array}$ & $\begin{array}{l}9.5 \\
3.1 \\
6.0 \\
1.1 \\
6.5 \\
1.6\end{array}$ & \\
\hline
\end{tabular}

Table 3: MCPP instances from Aldaya graph

\begin{tabular}{||l|l|l|l|l|l|r|l|r|r||}
\hline \hline & $p$ & $V_{R}$ & $E \cup A$ & LB0 & LBpol & Tpol & LB & Time & Dev (\%) \\
\hline albaR33 F1 & 24 & & & 10457 & 11300 & 1.8 & 11300 & 2.5 & 0.21 \\
albaR33 F2 & 24 & & & 10482 & 11300 & 1.5 & 11300 & 3.2 & 0.21 \\
albaR35 F1 & 30 & \multirow{2}{*}{116} & \multirow{2}{*}{174} & 13581.5 & 15710 & 0.6 & $15749^{*}$ & 1.3 & \\
albaR35 F2 & 30 & & & 15026 & 15710 & 0.4 & 15734 & 0.7 & 0.09 \\
albaR37 F1 & 24 & & & 11272 & 12982 & 1.0 & $13000^{*}$ & 1.0 & \\
albaR37 F2 & 24 & & & 11802 & 12982 & 0.7 & $13000^{*}$ & 1.0 & \\
\hline albaR53 F1 & 19 & & & 11489 & $12608^{*}$ & 1.4 & $12608^{*}$ & 1.1 & \\
albaR53 F2 & 19 & & & 11865 & $12608^{*}$ & 0.8 & $12608^{*}$ & 1.2 & \\
albaR55 F1 & 13 & 116 & \multirow{2}{*}{174} & 11038 & $12520^{*}$ & 1.0 & $12520^{*}$ & 0.9 & \\
albaR55 F2 & 13 & & & 11958 & $12520^{*}$ & 0.8 & $12520^{*}$ & 1.1 & \\
albaR57 F1 & 12 & & & 13369 & $15619^{*}$ & 2.6 & $15619^{*}$ & 2.7 & \\
albaR57 F2 & 12 & & & 14871 & $15619^{*}$ & 2.1 & $15619^{*}$ & 3.1 & \\
\hline albaR73 F1 & 8 & & & 13521 & $15301^{*}$ & 1.9 & $15301^{*}$ & 1.8 & \\
albaR73 F2 & 8 & & & 14875 & $15301^{*}$ & 0.9 & $15301^{*}$ & 1.3 & \\
albaR75 F1 & 2 & \multirow{2}{*}{116} & \multirow{2}{*}{174} & 13897 & 16550 & 2.2 & 16550 & 2.1 & 0.12 \\
albaR75 F2 & 2 & & & 15929 & 16550 & 1.1 & 16550 & 1.6 & 0.12 \\
albaR77 F1 & 5 & & & 13916.5 & $16358^{*}$ & 0.9 & $16358^{*}$ & 0.8 & \\
albaR77 F2 & 5 & & & 15639.5 & $16358^{*}$ & 0.7 & $16358^{*}$ & 0.9 & \\
\hline \hline
\end{tabular}

Table 4: MRPP instances from Albaida graph 


\begin{tabular}{|c|c|c|c|c|c|c|c|c|c|}
\hline & $p$ & $V_{R}$ & $E \cup A$ & LB0 & LBpol & Tpol & LB & Time & Dev (\%) \\
\hline madrR33 F1 & 49 & \multirow{6}{*}{196} & \multirow{6}{*}{316} & 18947.5 & $20570^{*}$ & 2.2 & $20570^{*}$ & 2.8 & \\
\hline madrR33 F2 & 49 & & & 19760 & $20570^{*}$ & 1.7 & $20570^{*}$ & 2.4 & \\
\hline madrR35 F1 & 48 & & & 21315 & $23100^{*}$ & 0.9 & $23100^{*}$ & 1.4 & \\
\hline madrR35 F2 & 48 & & & 22440 & $23100^{*}$ & 0.6 & $23100^{*}$ & 1.0 & \\
\hline madrR37 F1 & 39 & & & 24937.5 & $26740^{*}$ & 0.8 & $26740^{*}$ & 0.9 & \\
\hline madrR37 F2 & 39 & & & 26517.5 & $26740^{*}$ & 0.4 & $26740^{*}$ & 0.9 & \\
\hline madrR53 F1 & 25 & \multirow{6}{*}{196} & \multirow{6}{*}{316} & 19803.7 & $21255^{*}$ & 1.3 & $21255^{*}$ & 1.4 & \\
\hline madrR53 F2 & 25 & & & 20587.5 & $21255^{*}$ & 0.7 & $21255^{*}$ & 1.0 & \\
\hline madrR55 F1 & 19 & & & 26460 & $32390^{*}$ & 2.2 & $32390^{*}$ & 2.4 & \\
\hline madrR55 F2 & 19 & & & 32140 & $32390^{*}$ & 0.6 & $32390^{*}$ & 1.0 & \\
\hline madrR57 F1 & 20 & & & 30035 & $34455^{*}$ & 1.5 & $34455^{*}$ & 1.7 & \\
\hline madrR57 F2 & 20 & & & 34195 & $34455^{*}$ & 0.5 & $34455^{*}$ & 0.8 & \\
\hline madrR73 F1 & 5 & \multirow{6}{*}{196} & \multirow{6}{*}{316} & 23410 & 26047.5 & 11 & 26047.5 & 8.8 & 0.12 \\
\hline madrR73 F2 & 5 & & & 24947.5 & 26047.5 & 4.4 & 26047.5 & 5.7 & 0.12 \\
\hline madrR75 F1 & 4 & & & 30650 & $39235^{*}$ & 4.9 & $39235^{*}$ & 4.8 & \\
\hline madrR75 F2 & 4 & & & 38895 & $39235^{*}$ & 0.5 & $39235^{*}$ & 0.7 & \\
\hline madrR77 F1 & 5 & & & 29875 & $32470^{*}$ & 1.2 & $32470^{*}$ & 1.2 & \\
\hline madrR77 F2 & 5 & & & 31690 & $32470^{*}$ & 0.9 & $32470^{*}$ & 1.2 & \\
\hline
\end{tabular}

Table 5: MRPP instances from Madrigueras graph

\begin{tabular}{|c|c|c|c|c|c|c|c|c|c|}
\hline & $p$ & $V_{R}$ & $E \cup A$ & LB0 & LBpol & Tpol & LB & Time & Dev $(\%)$ \\
\hline aldaR33 F1 & 52 & \multirow{6}{*}{214} & \multirow{6}{*}{351} & 28782.8 & $31404^{*}$ & 4.6 & $31404^{*}$ & 7.4 & \multirow{6}{*}{$\begin{array}{l}0.03 \\
0.03\end{array}$} \\
\hline aldaR33 F2 & 52 & & & 30356.3 & $31404^{*}$ & 3.8 & $31404^{*}$ & 5.2 & \\
\hline aldaR35 F1 & 52 & & & 37264 & $41395^{*}$ & 1.0 & $41395^{*}$ & 1.7 & \\
\hline aldaR35 F2 & 52 & & & 40895 & $41395^{*}$ & 0.5 & $41395^{*}$ & 1.4 & \\
\hline aldaR37 F1 & 38 & & & 34410 & 37029 & 1.8 & 37029 & 2.7 & \\
\hline aldaR37 F2 & 38 & & & 35949 & 37029 & 1.4 & 37029 & 2.7 & \\
\hline aldaR53 F1 & 35 & \multirow{6}{*}{214} & \multirow{6}{*}{351} & 28506 & 30384.5 & 8.5 & $30392^{*}$ & 2.0 & \\
\hline aldaR53 F2 & 35 & & & 29501 & 30384.5 & 8.3 & $30392^{*}$ & 2.9 & \\
\hline aldaR55 F1 & 21 & & & 33849 & $36565^{*}$ & 2.4 & $36565^{*}$ & 2.3 & \\
\hline aldaR55 F2 & 21 & & & 35877 & $36565^{*}$ & 1.6 & $36565^{*}$ & 2.3 & \\
\hline aldaR57 F1 & 22 & & & 38425 & $42841^{*}$ & 1.4 & $42841^{*}$ & 1.5 & \\
\hline aldaR57 F2 & 22 & & & 42455 & $42841^{*}$ & 0.7 & $42841^{*}$ & 1.0 & \\
\hline aldaR73 F1 & 10 & \multirow{6}{*}{214} & \multirow{6}{*}{351} & 31610 & $34115^{*}$ & 3.9 & $34115^{*}$ & 3.0 & \\
\hline aldaR73 F2 & 10 & & & 33304 & $34115^{*}$ & 3.1 & $34115^{*}$ & 3.5 & \\
\hline aldaR75 F1 & 1 & & & 36610 & $40816^{*}$ & 6.1 & $40816^{*}$ & 6.0 & \\
\hline aldaR75 F2 & 1 & & & 39974 & $40816^{*}$ & 1.6 & $40816^{*}$ & 2.3 & \\
\hline aldaR77 F1 & 6 & & & 55006 & $64739^{*}$ & 3.7 & $64739^{*}$ & 3.7 & \\
\hline aldaR77 F2 & 6 & & & 64614 & $64739^{*}$ & 0.6 & $64739^{*}$ & 0.7 & \\
\hline
\end{tabular}

Table 6: MRPP instances from Aldaya graph 


\begin{tabular}{||l|l|l|l|l|l|r|l|r|r||}
\hline \hline & $p$ & $V_{R}$ & $E \cup A$ & LB0 & LBpol & Tpol & LB & Time & Dev (\%) \\
\hline albaG33 F1 & 66 & & & 10120 & 11792.7 & 1.2 & $11796^{*}$ & 2.2 & \\
albaG33 F2 & 66 & & & 10272 & 11792.7 & 0.9 & $11796^{*}$ & 2.4 & \\
albaG35 F1 & 68 & \multirow{2}{*}{116} & \multirow{2}{*}{174} & 8890 & 11146 & 2.1 & 11166 & 2.9 & 0.16 \\
albaG35 F2 & 68 & & & 9075 & 11146 & 1.5 & 11166 & 3.0 & 0.16 \\
albaG37 F1 & 72 & & & 14613 & 17583 & 0.7 & 17651.6 & 2.2 & 0.06 \\
albaG37 F2 & 72 & & & 14757 & 17583 & 0.7 & 17651 & 3.0 & 0.06 \\
\hline albaG53 F1 & 35 & & & 10107 & $11551^{*}$ & 0.6 & $11551^{*}$ & 0.6 & \\
albaG53 F2 & 35 & & & 10595 & $11551^{*}$ & 0.6 & $11551^{*}$ & 1.0 & \\
albaG55 F1 & 28 & \multirow{2}{*}{116} & \multirow{2}{*}{174} & 13357 & 14393 & 0.7 & 14445 & 0.7 & 0.14 \\
albaG55 F2 & 28 & & & 13915 & 14393 & 0.5 & 14445 & 0.7 & 0.14 \\
albaG57 F1 & 34 & & & 15475 & $17124^{*}$ & 1.3 & $17124^{*}$ & 1.3 & \\
albaG57 F2 & 34 & & & 16406 & $17124^{*}$ & 0.9 & $17124^{*}$ & 1.5 & \\
\hline albaG73 F1 & 13 & & & 12550 & $14030^{*}$ & 1.0 & $14030^{*}$ & 1.0 & \\
albaG73 F2 & 13 & & & 13142 & $14030^{*}$ & 0.5 & $14030^{*}$ & 0.7 & \\
albaG75 F1 & 12 & 116 & \multirow{2}{*}{174} & 14235 & $16109^{*}$ & 1.3 & $16109^{*}$ & 1.3 & \\
albaG75 F2 & 12 & & & 15271 & $16109^{*}$ & 0.7 & $16109^{*}$ & 0.8 & \\
albaG77 F1 & 7 & & & 16052 & $18182^{*}$ & 1.3 & $18182^{*}$ & 1.2 & \\
albaG77 F2 & 7 & & & 17458 & $18182^{*}$ & 0.9 & $18182^{*}$ & 1.3 & \\
\hline \hline
\end{tabular}

Table 7: MGRP instances from Albaida graph

\begin{tabular}{|c|c|c|c|c|c|c|c|c|c|}
\hline & $p$ & $V_{R}$ & $E \cup A$ & LB0 & LBpol & Tpol & LB & Time & Dev (\%) \\
\hline madrG33 F1 & 104 & \multirow{6}{*}{196} & \multirow{6}{*}{316} & 16740 & 17573.7 & 5.8 & 17631.7 & 112 & 0.83 \\
\hline madrG33 F2 & 104 & & & 16915 & 17573.7 & 4.8 & 17623.2 & 79 & 0.88 \\
\hline madrG35 F1 & 97 & & & 20425 & 21675 & 1.5 & 21735 & 5.3 & 0.30 \\
\hline madrG35 F2 & 97 & & & 21020 & 21675 & 0.6 & 21735 & 6.0 & 0.30 \\
\hline madrG37 F1 & 99 & & & 22555 & 23830 & 0.5 & 23853.3 & 2.5 & 0.03 \\
\hline madrG37 F2 & 99 & & & 23110 & 23830 & 0.3 & 23853.3 & 2.6 & 0.03 \\
\hline madrG53 F1 & 44 & \multirow{6}{*}{196} & \multirow{6}{*}{316} & 20235 & 21747.5 & 8.8 & $21785^{*}$ & 2.2 & \multirow{6}{*}{$\begin{array}{l}0.06 \\
0.05\end{array}$} \\
\hline madrG53 F2 & 44 & & & 20822.5 & 21747.5 & 4.8 & $21785^{*}$ & 1.8 & \\
\hline madrG55 F1 & 45 & & & 23437.5 & 26301.2 & 1.8 & 26338.7 & 3.5 & \\
\hline madrG55 F2 & 45 & & & 25695 & 26301.2 & 0.8 & 26342.5 & 3.0 & \\
\hline madrG57 F1 & 41 & & & 25070 & $27700^{*}$ & 2.1 & $27700^{*}$ & 2.9 & \\
\hline madrG57 F2 & 41 & & & 27600 & $27700^{*}$ & 0.8 & $27700^{*}$ & 1.7 & \\
\hline madrG73 F1 & 14 & \multirow{6}{*}{196} & \multirow{6}{*}{316} & 23297.5 & $26365^{*}$ & 3.9 & $26365^{*}$ & 4.0 & \\
\hline madrG73 F2 & 14 & & & 25707.5 & $26365^{*}$ & 2.3 & $26365^{*}$ & 4.6 & \\
\hline madrG75 F1 & 7 & & & 27315 & 33320 & 3.7 & $33355^{*}$ & 4.1 & \\
\hline madrG75 F2 & 7 & & & 32697.5 & 33320 & 0.7 & $33355^{*}$ & 1.0 & \\
\hline madrG77 F1 & 10 & & & 32420 & $35405^{*}$ & 2.4 & $35405^{*}$ & 2.4 & \\
\hline madrG77 F2 & 10 & & & 35031.2 & $35405^{*}$ & 0.9 & $35405^{*}$ & 1.2 & \\
\hline
\end{tabular}

Table 8: MGRP instances from Madrigueras graph 


\begin{tabular}{|c|c|c|c|c|c|c|c|c|c|}
\hline & $p$ & $V_{R}$ & $E \cup A$ & LB0 & LBpol & Tpol & $\mathrm{LB}$ & Time & $\operatorname{Dev}(\%)$ \\
\hline aldaG33 F1 & 109 & \multirow{6}{*}{214} & \multirow{6}{*}{351} & 24178.5 & 24980.7 & 5.3 & 25050.5 & 29 & 0.64 \\
\hline aldaG33 F2 & 109 & & & 24231 & 24980.7 & 3.1 & 25050.5 & 21 & 0.64 \\
\hline aldaG35 F1 & 100 & & & 28842 & 31446 & 1.9 & 31652 & 11 & 0.66 \\
\hline aldaG35 F2 & 100 & & & 29982 & 31446 & 1.6 & 31652 & 13 & 0.66 \\
\hline aldaG37 F1 & 105 & & & 28315 & 31480 & 1.3 & 31538 & 9.8 & 0.01 \\
\hline aldaG37 F2 & 105 & & & 28919 & 31480 & 1.0 & 31538 & 9.7 & 0.01 \\
\hline aldaG53 F1 & 39 & \multirow{6}{*}{214} & \multirow{6}{*}{351} & 32146 & 36197 & 7.1 & $36213^{*}$ & 10 & \multirow{6}{*}{0.01} \\
\hline aldaG53 F2 & 39 & & & 35286 & 36197 & 4.1 & 36209 & 9.0 & \\
\hline aldaG55 F1 & 51 & & & 33055 & $36903^{*}$ & 2.4 & $36903^{*}$ & 2.5 & \\
\hline aldaG55 F2 & 51 & & & 36017.5 & $36903^{*}$ & 1.0 & $36903^{*}$ & 2.0 & \\
\hline aldaG57 F1 & 35 & & & 38366.5 & $40832^{*}$ & 1.4 & $40832^{*}$ & 1.9 & \\
\hline aldaG57 F2 & 35 & & & 40611 & $40832^{*}$ & 0.5 & $40832^{*}$ & 1.2 & \\
\hline aldaG73 F1 & 9 & \multirow{6}{*}{214} & \multirow{6}{*}{351} & 34020 & $36995^{*}$ & 4.1 & $36995^{*}$ & 3.5 & \\
\hline aldaG73 F2 & 9 & & & 35956 & $36995^{*}$ & 1.3 & $36995^{*}$ & 2.9 & \\
\hline aldaG75 F1 & 6 & & & 39761 & $47228^{*}$ & 6.4 & $47228^{*}$ & 6.2 & \\
\hline aldaG75 F2 & 6 & & & 46329 & $47228^{*}$ & 3.6 & $47228^{*}$ & 4.8 & \\
\hline aldaG77 F1 & 10 & & & 38655 & $41730^{*}$ & 1.5 & $41730^{*}$ & 1.6 & \\
\hline aldaG77 F2 & 10 & & & 41197 & $41730^{*}$ & 0.7 & $41730^{*}$ & 0.9 & \\
\hline
\end{tabular}

Table 9: MGRP instances from Aldaya graph 


\begin{tabular}{|c|c|c|c|c|c|c|c|c|c|}
\hline & $p$ & $V_{R}$ & $E \cup A$ & LB0 & LBpol & Tpol & LB & Time & Dev $(\%)$ \\
\hline $\begin{array}{ll}\mathrm{R} 422 & \mathrm{~F} 1 \\
\mathrm{R} 422 & \mathrm{~F} 2 \\
\mathrm{R} 425 & \mathrm{~F} 1 \\
\mathrm{R} 425 & \mathrm{~F} 2 \\
\mathrm{R} 427 & \mathrm{~F} 1 \\
\mathrm{R} 427 & \mathrm{~F} 2\end{array}$ & 102 & 357 & 884 & $\begin{array}{l}18005.5 \\
18099 \\
20828 \\
21404 \\
29328 \\
30242.5\end{array}$ & $\begin{array}{l}18992.6 \\
18992.6 \\
22211.5 \\
22211.5 \\
30615 \\
30615\end{array}$ & $\begin{array}{r}14.7 \\
15.8 \\
3.9 \\
4.3 \\
1.9 \\
2.2\end{array}$ & $\begin{array}{l}19009.7 \\
19009.3 \\
22234^{*} \\
22234^{*} \\
30615 \\
30615\end{array}$ & $\begin{array}{r}45.1 \\
120 \\
11.0 \\
12.7 \\
8.2 \\
10.0\end{array}$ & $\begin{array}{l}0.11 \\
0.11\end{array}$ \\
\hline $\begin{array}{l}\text { R452 F1 } \\
\text { R452 F2 } \\
\text { R455 F1 } \\
\text { R455 F2 } \\
\text { R457 F1 } \\
\text { R457 F2 }\end{array}$ & 23 & 465 & 1055 & $\begin{array}{l}27321 \\
28264 \\
33112.2 \\
34916.3 \\
45038 \\
48441\end{array}$ & $\begin{array}{l}29359.5 \\
29359.5 \\
35862.5 \\
35862.5 \\
49155 \\
49155\end{array}$ & $\begin{array}{r}726 \\
3042 \\
25.3 \\
24.9 \\
26.2 \\
12.5\end{array}$ & $\begin{array}{l}29359.5 \\
29359.5 \\
35862.5 \\
35862.5 \\
49162^{*} \\
49162^{*}\end{array}$ & $\begin{array}{r}716 \\
426 \\
28.8 \\
27.3 \\
22.6 \\
13.5\end{array}$ & $\begin{array}{l}0.01 \\
0.01 \\
0.09 \\
0.09\end{array}$ \\
\hline $\begin{array}{l}\text { R472 F1 } \\
\text { R472 F2 } \\
\text { R475 F1 } \\
\text { R475 F2 } \\
\text { R477 F1 } \\
\text { R477 F2 }\end{array}$ & 3 & 498 & 1114 & $\begin{array}{l}37642.5 \\
39839 \\
43872.5 \\
49174.5 \\
59846.5 \\
66068\end{array}$ & $\begin{array}{l}40744^{*} \\
40744^{*} \\
49972^{*} \\
49972^{*} \\
66481^{*} \\
66481^{*}\end{array}$ & $\begin{array}{r}328 \\
188 \\
111 \\
9.8 \\
23.9 \\
4.3\end{array}$ & $\begin{array}{l}40744^{*} \\
40744^{*} \\
49972^{*} \\
49972^{*} \\
66481^{*} \\
66481^{*}\end{array}$ & $\begin{array}{r}304 \\
91.6 \\
109 \\
9.7 \\
23.7 \\
4.1\end{array}$ & \\
\hline $\begin{array}{l}\text { R522 F1 } \\
\text { R522 F2 } \\
\text { R525 F1 } \\
\text { R525 F2 } \\
\text { R527 F1 } \\
\text { R527 F2 }\end{array}$ & 79 & 388 & 1106 & $\begin{array}{l}21249.7 \\
21429 \\
25537.3 \\
25965.8 \\
33867 \\
35231.5\end{array}$ & $\begin{array}{l}22287.6 \\
22287.6 \\
26669^{*} \\
26669^{*} \\
36003.5 \\
36003.5\end{array}$ & $\begin{array}{r}76.8 \\
273 \\
26.1 \\
75.8 \\
2.6 \\
3.9\end{array}$ & $\begin{array}{l}22292.5 \\
22292.5 \\
26669^{*} \\
26669^{*} \\
36038.4 \\
36038.2\end{array}$ & $\begin{array}{r}135 \\
105 \\
92.4 \\
64.1 \\
46.0 \\
44.0\end{array}$ & $\begin{array}{l}0.12 \\
0.12\end{array}$ \\
\hline $\begin{array}{l}\text { R552 F1 } \\
\text { R552 F2 } \\
\text { R555 F1 } \\
\text { R555 F2 } \\
\text { R557 F1 } \\
\text { R557 F2 }\end{array}$ & 7 & 488 & 1318 & $\begin{array}{l}34343.5 \\
34895.2 \\
40408.6 \\
42217.5 \\
54120 \\
56745.5\end{array}$ & $\begin{array}{l}35958^{*} \\
35958^{*} \\
42951 \\
42951 \\
57096^{*} \\
57096^{*}\end{array}$ & $\begin{array}{r}757 \\
4106 \\
32.6 \\
245 \\
8.0 \\
5.8\end{array}$ & $\begin{array}{l}35958^{*} \\
35958^{*} \\
42951 \\
42951 \\
57096^{*} \\
57096^{*}\end{array}$ & $\begin{array}{r}541 \\
672 \\
33.4 \\
99.0 \\
8.4 \\
6.0\end{array}$ & $\begin{array}{l}0.01 \\
0.01\end{array}$ \\
\hline $\begin{array}{l}\text { R572 F1 } \\
\text { R572 F2 } \\
\text { R575 F1 } \\
\text { R575 F2 } \\
\text { R577 F1 } \\
\text { R577 F2 }\end{array}$ & 1 & 498 & 1326 & $\begin{array}{l}48479 \\
49261.9 \\
53508 \\
56971.5 \\
65212.5 \\
71267.5\end{array}$ & $\begin{array}{l}50304^{*} \\
50304^{*} \\
57880^{*} \\
57880^{*} \\
71769^{*} \\
71769^{*}\end{array}$ & $\begin{array}{r}68.6 \\
30.2 \\
362 \\
28.9 \\
60.1 \\
12.6\end{array}$ & $\begin{array}{l}50304^{*} \\
50304^{*} \\
57880^{*} \\
57880^{*} \\
71769^{*} \\
71769^{*}\end{array}$ & $\begin{array}{r}68.9 \\
40.6 \\
365 \\
25.1 \\
61.5 \\
11.9\end{array}$ & \\
\hline
\end{tabular}

Table 10: Random MRPP instances 


\begin{tabular}{|c|c|c|c|c|c|c|c|c|c|}
\hline & $p$ & $V_{R}$ & $E \cup A$ & LB0 & LBpol & Tpol & $\mathrm{LB}$ & Time & $\operatorname{Dev}(\%)$ \\
\hline G422 F1 & \multirow{6}{*}{245} & \multirow{6}{*}{500} & \multirow{6}{*}{1105} & 20536 & 21934.3 & 36.7 & 21990.8 & 2384 & 0.62 \\
\hline G422 F2 & & & & 20552.7 & 21934.3 & 72.3 & 21987.5 & 4964 & 0.63 \\
\hline G425 F1 & & & & 23520.5 & 25793.3 & 20.4 & 25827.6 & 731 & 0.41 \\
\hline $\mathrm{G} 425 \mathrm{~F} 2$ & & & & 24057 & 25793.3 & 26.4 & 25827.5 & 588 & 0.41 \\
\hline G427 F1 & & & & 27252 & 29492 & 13.8 & 29513 & 699 & 0.31 \\
\hline G427 F2 & & & & 27572 & 29492 & 20.4 & 29517.2 & 731 & 0.30 \\
\hline G452 F1 & \multirow{6}{*}{58} & \multirow{6}{*}{500} & \multirow{6}{*}{1118} & 29829 & 32667 & 36.6 & $32672^{*}$ & 30.4 & \multirow{6}{*}{$\begin{array}{l}0.05 \\
0.05\end{array}$} \\
\hline G452 F2 & & & & 31362.5 & 32667 & 13.5 & $32672^{*}$ & 21.4 & \\
\hline G455 F1 & & & & 33118.7 & 35963.5 & 189 & 35998.6 & 118 & \\
\hline G455 F2 & & & & 34951.1 & 35963.5 & 436 & 35998.6 & 183 & \\
\hline G457 F1 & & & & 40666.5 & 43025 & 6.6 & $43035^{*}$ & 11.0 & \\
\hline G457 F2 & & & & 42352 & 43025 & 4.3 & $43035^{*}$ & 8.0 & \\
\hline G472 F1 & \multirow{6}{*}{5} & \multirow{6}{*}{500} & \multirow{6}{*}{1116} & 37471.5 & 39725 & 974 & 39725 & 251 & \multirow{6}{*}{$\begin{array}{l}0.00 \\
0.00\end{array}$} \\
\hline G472 F2 & & & & 38601.5 & 39725 & 1932 & 39725 & 788 & \\
\hline G475 F1 & & & & 42556.5 & $50307^{*}$ & 75.9 & $50307^{*}$ & 71.1 & \\
\hline G475 F2 & & & & 49573.5 & $50307^{*}$ & 15 & $50307^{*}$ & 17.2 & \\
\hline G477 F1 & & & & 52060.5 & $57952^{*}$ & 21.4 & $57952^{*}$ & 22.6 & \\
\hline G477 F2 & & & & 57374 & $57952^{*}$ & 16.6 & $57952^{*}$ & 14.3 & \\
\hline G522 F1 & \multirow{6}{*}{191} & \multirow{6}{*}{500} & \multirow{6}{*}{1301} & 22762 & 24372.0 & 206 & 24490.3 & 3734 & 0.55 \\
\hline G522 F2 & & & & 22963.5 & 24371.9 & 129 & 24478.5 & 6480 & 0.59 \\
\hline G525 F1 & & & & 25729.5 & 26602.2 & 10.7 & 26651.2 & 183 & 0.32 \\
\hline G525 F2 & & & & 25829.2 & 26602.2 & 11.5 & 26650.5 & 219 & 0.32 \\
\hline G527 F1 & & & & 31177 & 32774.5 & 8.8 & 32802 & 129 & 0.08 \\
\hline G527 F2 & & & & 31485 & 32774.5 & 7.3 & 32802 & 114 & 0.08 \\
\hline G552 F1 & \multirow{6}{*}{19} & \multirow{6}{*}{500} & \multirow{6}{*}{1339} & 34683 & $36394^{*}$ & 411 & $36394^{*}$ & 230 & \multirow{6}{*}{$\begin{array}{l}0.00 \\
0.00\end{array}$} \\
\hline G552 F2 & & & & 35464.5 & $36394^{*}$ & 85.6 & $36394^{*}$ & 88.3 & \\
\hline G555 F1 & & & & 41229.5 & 43243.5 & 19.3 & 43268 & 19.2 & \\
\hline G555 F2 & & & & 42573.8 & 43243.5 & 8.8 & 43268 & 7.9 & \\
\hline G557 F1 & & & & 54019 & $57414^{*}$ & 14.7 & $57414^{*}$ & 14.7 & \\
\hline G557 F2 & & & & 57084 & $57414^{*}$ & 6.2 & $57414^{*}$ & 6.5 & \\
\hline G572 F1 & \multirow{6}{*}{3} & \multirow{6}{*}{500} & \multirow{6}{*}{1329} & 48360.5 & $50058^{*}$ & 1787 & $50058^{*}$ & 609 & \\
\hline G572 F2 & & & & 48908.5 & $50058^{*}$ & 217 & $50058^{*}$ & 112 & \\
\hline G575 F1 & & & & 53296 & $61128^{*}$ & 1384 & $61128^{*}$ & 1033 & \\
\hline G575 F2 & & & & 60242 & $61128^{*}$ & 82.5 & $61128^{*}$ & 48.2 & \\
\hline G577 F1 & & & & 71266 & 79352 & 79.6 & $79359^{*}$ & 87.6 & \\
\hline G577 F2 & & & & 79123.5 & 79352 & 5.4 & $79359^{*}$ & 6.9 & \\
\hline
\end{tabular}

Table 11: Random MGRP instances 